\title{
结合通道空间加权特征金字塔网络的白细胞检测与分割
}

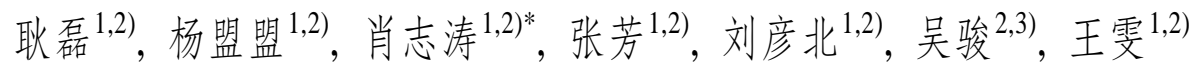 \\ ${ }^{1)}$ (天津工业大学生命科学学院 天津 300387) \\ ${ }^{2)}$ (天津市光电检测技术与系统重点实验室 天津 300387) \\ 3) (天津工业大学电子与信息工程学院 天津 300387) \\ (xiaozhitao@tiangong.edu.cn)
}

\begin{abstract}
摘 要: 白细胞的准确检测和精确分割是一项具有挑战性的医学图像处理任务. 在显微镜下获取的白细胞图像会受 到染色杂质的影响, 且白细胞种类繁多、形态各异、类间差别小, 还存在相互重叠相互粘连的现象, 导致细胞边缘无 法被准确分割，上述问题一直都是白细胞图像检测和分割的难点. 针对以上问题，基于 Mask R-CNN 提出了结合注 意力机制多尺度特征融合的白细胞检测方法. 在 Mask R-CNN 结构的基础上, 在特征金字塔网络(FPN)模块中融合了 注意力机制模块, 提出了通道空间加权特征金字塔网络. 该结构不仅可以学习特征图中重要通道特征的权重大小, 还可以学习层中重要特征区域的表示. 同时, 在网络结构中加人了 Skip-FPN 模块, 该模块通过短连接融合更多白细 胞的底层细节信息, 从而更准确地检测白细胞, 更精确地进行白细胞的形态分割. 实验结果表明, 所提方法具有良好 的检测与分割性能. 在 Kaggle 开源数据集下, 所提方法对白细胞检测的指标 mAP 值达到了 98.25\%, 与改进前相比提 高了 $1.25 \%$; 分割的平均精度 mIoU 值达到了 $89.30 \%$, 与改进前相比提高了 $0.002 \%$.
\end{abstract}

关键词: 卷积神经网络; 语义分割; 多尺度特征; 通道空间加权

中图法分类号: TP391.41ＤOI: 10.3724/SP.J.1089.2021.18672

\section{White Blood Cell Detection and Segmentation Combined with the Channel Space Weighted Feature Pyramid Networks}

\author{
Geng Lei ${ }^{1,2)}$, Yang Mengmeng ${ }^{1,2)}$, Xiao Zhitao ${ }^{1,2)^{*}}$, Zhang Fang ${ }^{1,2)}$, Liu Yanbei ${ }^{1,2)}$, Wu Jun ${ }^{2,3)}$, and Wang Wen ${ }^{1,2)}$ \\ ${ }^{1)}$ (School of Life Sciences, Tiangong University, Tianjin 300387) \\ ${ }^{2)}$ (Tianjin Key Laboratory of Optoelectronic Detection Technology and System, Tianjin 300387) \\ ${ }^{3)}$ (School of Electronics and Information Engineering, Tiangong University, Tianjin 300387)
}

\begin{abstract}
The accurate detection and segmentation of leukocytes is a challenging task in medical image processing. The white blood cell image obtained under microscope is easily affected by impurities. There are many kinds of white cells, different shapes and small differences among them, and also exiting overlapping and adhesion phenomena, which leads to the inaccuracy of cell edge segmentation. The above problems are always the difficulties of detection and segmentation of white cell image. To solve the above problems, a leukocyte detection method based on Mask R-CNN and attention mechanism multi-scale feature fusion is proposed. Based on the Mask R-CNN structure, proposed thesis integrates the attention mechanism module in the FPN (feature pyramid networks) module, and proposes the CSFPN (channel spatial feature pyramid

收稿日期: 2020-09-20; 修回日期: 2021-06-02. 基金项目: 天津市科技重大专项与工程(17ZXSCSY00060, 17ZXHLSY00040). 耿磊(1982一), 男, 博士, 副教授, 硕士生导师, 主要研究方向为图像处理、深度学习; 杨盟盟(1995一), 女, 硕士研究生, 主要研究方 向为图像处理; 肖志涛(1971一), 男, 博士, 教授, 博士生导师, 论文通讯作者, 主要研究方向为智能信号处理; 张芳(1981一), 女, 博士, 教授, 硕士生导师, 主要研究方向为图像处理、模式识别; 刘彦北(1985一), 男, 博士, 讲师, 主要研究方向为图像处理、深度 学习; 吴骏(1978一), 男, 博士, 副教授, 硕士生导师, 主要研究方向为图像处理; 王雯(1990一), 女, 博士, 助教, 主要研究方向为
\end{abstract} 图像处理. 
networks) structure. This structure can learn the weight of important channel features and the representation of important feature regions in the feature maps. At the same time, Skip-FPN module is added to the network structure, which fuses more low-level detailed information of leukocytes through short connection, so as to detect and segment leukocytes more accurately. Experimental results show that this method has good detection and segmentation performance. Under the Kaggle open source data set, the mAP value of this method for white blood cell detection reaches $98.25 \%$, which has an increase of $1.25 \%$ compared with the previous improvement. The accuracy mIoU value reached $89.3 \%$, which has an increase of $0.002 \%$ compared to the before improvement.

Key words: convolutional neural network; semantic segmentation; multi-scale features; channel space weighting

血液疾病会导致外周血中各类白细胞的总数 和百分比发生变化、各类白细胞的形态出现增大、 细胞核的数量增多或细胞核的形态呈不规则形状 等问题. 外周血有核细胞的显微镜检查是白血病、 贫血等血液疾病的基本诊断方法之一. 目前, 自动 化血细胞分析仪已是国内外临床检验最常用的篮 选仪器之一，应用多项检测原理对各项血细胞检 测参数进行分析检测, 为临床不同层次需求提供 更有效、精确的白细胞检测参数, 但是自动化血细 胞分析仪不能检测白细胞的形态变化，因此需要 医生进行人工镜检. 医学专家在沾有血液的载玻 片上计数 100 或 200 个白细胞, 并依据计数结果计 算每种白细胞的百分比 ${ }^{[1]}$. 在用显微镜肉眼观察 时，医生必须具备丰富的经验，而人工镜检比较耗 时、工作量大、乏味且统计量小, 容易有主观判断. 在人工镜检过程中，医生主要通过计数各类白细 胞和观察白细胞的形态进行血液疾病的诊断, 因 此利用机器学习对白细胞进行检测和形态学的分 割对血液疾病诊断具有重要意义.

对白细胞图像进行检测和分割不仅需要专业 知识, 而且需要具备丰富的医学经验, 因此显微镜 下检测和分割白细胞很困难。随着血液病计算机 辅助诊断系统的发展, 白细胞的自动检测技术也 在不断发展. 国内外已经提出了大量的白细胞检 测和分割算法, 根据训练方法不同, 可分为无监督 和有监督 2 类算法.

无监督算法不需要对白细胞做标签，根据不 同的处理过程又可以细分为基于聚类分割算法 ${ }^{[2-5]}$ 和基于國值分割算法 ${ }^{[6-7]}$. 基于聚类的白细胞分割 算法以欧几里得度量作为相似度，基于图像像素 值进行聚类获得分割结果。该算法过分依赖初始 化数据和其他参数, 聚类结果与目标数据的数量 和形状密切相关. 当细胞质的颜色与细胞核的颜
色显著不同时，该策略无法精确地获得所有白细 胞. 基于國值的白细胞分割算法是利用白细胞与 背景在灰度上的差异，选择一个合适的阈值，通过 判断每个像素点的特征属性进行白细胞的分割. 当白细胞相互重叠时, 该策略无法获得精确的白 细胞分割形态，且在处理过程中容易丢失信息. Liu 等 ${ }^{[2]}$ 提出了一种快速、准确的骨髓细胞分割算 法，先使用聚类和分水岭算法去除骨髓显微图像 中的杂质, 再通过 $K$-means 聚类方法选择候选掩 码, 并通过分水岭算法对相连的细胞进行分割. Duan 等 ${ }^{6]}$ 将基于颜色直方图的分割算法与基于区 域增长合并的分割算法相结合, 提出了一种饱和 阈值的彩色图像分割算法. Cao 等 ${ }^{[7]}$ 提出了一种通 过分割细胞核与细胞质对外周血的白细胞进行分 割的算法, 细胞核采用平均分离法进行分割, 细胞 质通过最小化模糊散度进行分割. 黄震等 ${ }^{[8]}$ 提出一 种在白细胞检测的基础之上进行白细胞分割的算 法, 首先通过白细胞检测的方法获得白细胞的位 置, 得到只包括白细胞的图像; 然后采用分割算法 对得到的白细胞图像再进行分割, 可以避免背景 中红细胞和血小板的影响. 杨小青等 ${ }^{[9]}$ 提出了采用 数学形态学对白细胞图像进行分割的算法, 有效 地避免了使用显微镜带来的光照不均匀和对血涂 片染色带来的图像斑点的干扰, 但是会出现过度 分割的问题. 当白细胞的形态、颜色和大小变化很 大且需要调整参数防止过度分割时, 无监督算法 的分割效果不佳. 而且无监督算法都采用了某种 结构，每个方法都是针对具体应用，不一定适合所 有的细胞数据集，泛化能力和鲁棒性较弱.

有监督算法将分割建模为多类分类问题，传 统方法主要包括神经网络、 $K$ 近邻分类器、支持向 量机(support vector machine, SVM)等. Mohammadi 等 ${ }^{[10]}$ 提出通过卷积神经网络(convolutional neural 
networks, CNN) 模型和高斯混合模型 (Gaussian mixture model, GMM)对血涂片图像中的白细胞进 行快速、准确分割的方法. Loddo 等 ${ }^{[11]}$ 提出了一种 利用近邻思想和支持向量机技术进行细胞分割的 方法. 这些传统方法的分类模型依赖于手动特征 选取的好坏, 并且需要大量的预先分割好的白细 胞图像作为训练样本保证模型的精确度, 对医学 图像要求比较高.

近年来, 结合 $K$-means 聚类和 SVM 分类器的 自我监督学习方法被应用于白细胞分割中. Razzak 等 ${ }^{[12]}$ 提出了一种基于完全常规网络的有效轮廓感 知分割方法, 并基于从每个分割单元中提取的 $\mathrm{CNN}$ 特征, 使用机器学习进行分类. Qin 等 ${ }^{[13]}$ 提出 了一种根据白血病测试领域的知识, 构建基于深 度残差学习理论的白细胞分类器的方案, 测试集 的平均准确率接近 $76.84 \%$. 以上方法存在一种普 遍的局限性, 即基于某些先验知识手动设计特征 和调整参数, 这样会导致特征提取不充分, 从而导 致网络检测性能差、分割结果差等问题, 所以上述 方法不适用于白细胞的检测和分割.

近年来, 深度学习算法取得了关键突破, 学习 方式由最初的浅层特征到进一步的深层特征, 很 好地解决了传统图像处理方法中遇到的很多问题. 深度学习根据大量样本的学习能够得到深层的、数 据集特定的特征表示，对数据集的表达更高效和 准确, 所提取的抽象特征鲁棒性更强, 泛化能力更 好. Tiwari 等 ${ }^{[14]}$ 提出了一种深度学习模型, 基于 $\mathrm{CNN}$ 框架自动对血细胞图像进行分类. $\mathrm{Yu}$ 等 ${ }^{[15]}$ 提 出了一种使用多种网络组合而形成的模型, 能够 在短时间内高精度地对不同种类的白细胞进行分 类. Tran 等 ${ }^{[16]}$ 提出利用 SegNet 对红细胞与白细胞 进行分割, 整体精度为 $89.45 \%$. Zhang 等 ${ }^{[17]}$ 提出了 使用 U-Net 进行白细胞分割, 并建立 CapsNet 模型 进行白细胞的预测与分类, 白细胞图像测试集的分 类精度为 $85.00 \%$. Wang 等 ${ }^{[18]}$ 提出使用 YOLO(you only look once)和 SSD(single shot multibox detector)的网络框架对 11 类白细胞进行检测, 平均精度 为 $90.09 \%$. 上述基于 CNN 的方法均在整个图像上 分割细胞, 分割结果易受背景杂质的影响. 由于白 细胞图像中含有大量染色杂质, 因此上述基于 CNN 的方法无法满足准确分割血涂片中白细胞的 要求.

Mask R-CNN ${ }^{[19]}$ 在 Faster R-CNN ${ }^{[20]}$ 基础之上 进行扩展, 并行地在边框回归分支上添加一个用 于预测目标掩码的新分支, 将掩码预测和分类预
测拆解. 除此之外, Mask R-CNN 还提出了感兴趣 区域(region of interest, ROI)Align, 解决了 ROI Pooling 在运算时存在定位误差的问题. 并且, Mask R-CNN 在实现检测任务的同时对每个目标 输出高质量的分割结果. 因此, 该多任务网络模型 适用于本文研究对象的检测和分割.

白细胞的检测和分割一直被社会界广泛关注， 其算法一直在逐步完善. 目前, 白细胞检测和分割 所面临的难点如下：(1) 细胞之间相互重叠、相互 粘连; (2) 白细胞边界特征不明显; (3) 显微镜下获 取的图像会受到不同的染色方法、染色程度和光照 条件的影响; (4) 白细胞种类繁多, 形态多变, 具 有复杂多样性, 类间差别小, 分类难度高. 解决这 些难点的关键步骤如下：(1) 提取完整的细胞形态 特征; (2) 提取有效细胞边缘信息进行细胞边缘的 精确定位; (3) 抑制杂质特征的提取; (4) 提取有效 语义信息进行白细胞精确分类.

结合文献[21-22], 考虑白细胞图像中存在杂 质、白细胞边界特征不明显且类间差别小等难点, 本文提出了一种端到端的白细胞检测和分割方法, 可以从血涂片图像中准确检测出白细胞对其进行 精确分割, 并可以从白细胞数量、形态方面对白细 胞进行分析和诊断. 本文主要研究白细胞的检测 与分割内容, 结合不同种类的白细胞特征之间的 极度相似性和白细胞边缘特征模糊的特性, 提出 了结合通道空间加权与跳跃连接的特征融合模块. 通道空间加权模块使网络按照重要程度自适应调 整各通道和空间的特征响应, 完成特征的重新标 定; 跳跃连接模块主要目的是缩短特征从底层到 顶层的传递路径，融合更多白细胞细节信息.

\section{1 基础网络}

\subsection{Mask R-CNN 模型}

本文选用 Mask R-CNN 作为基准检测模型, 基本框架如图 1 所示. 此前基于深度学习的目标检 测流程包括选择候选区域、提取特征、分类与边界 回归. Mask R-CNN 模型主要由 CNN、区域建议网 络(region proposal networks, RPN)和 3 个分支网络 (定位、分类、语义分割)构成.

\subsection{ResNet-50}

本文使用 ResNet- $50^{[23]}$ 作为基础网络提取白细 胞特征, ResNet-50 是由 50 个基础残差块组成, 基 础残差块的结构如图 2 所示. 其中, 输人为 $x$, 期 望的输出为 $H(x)$, 残差块的学习目标为输出和输 


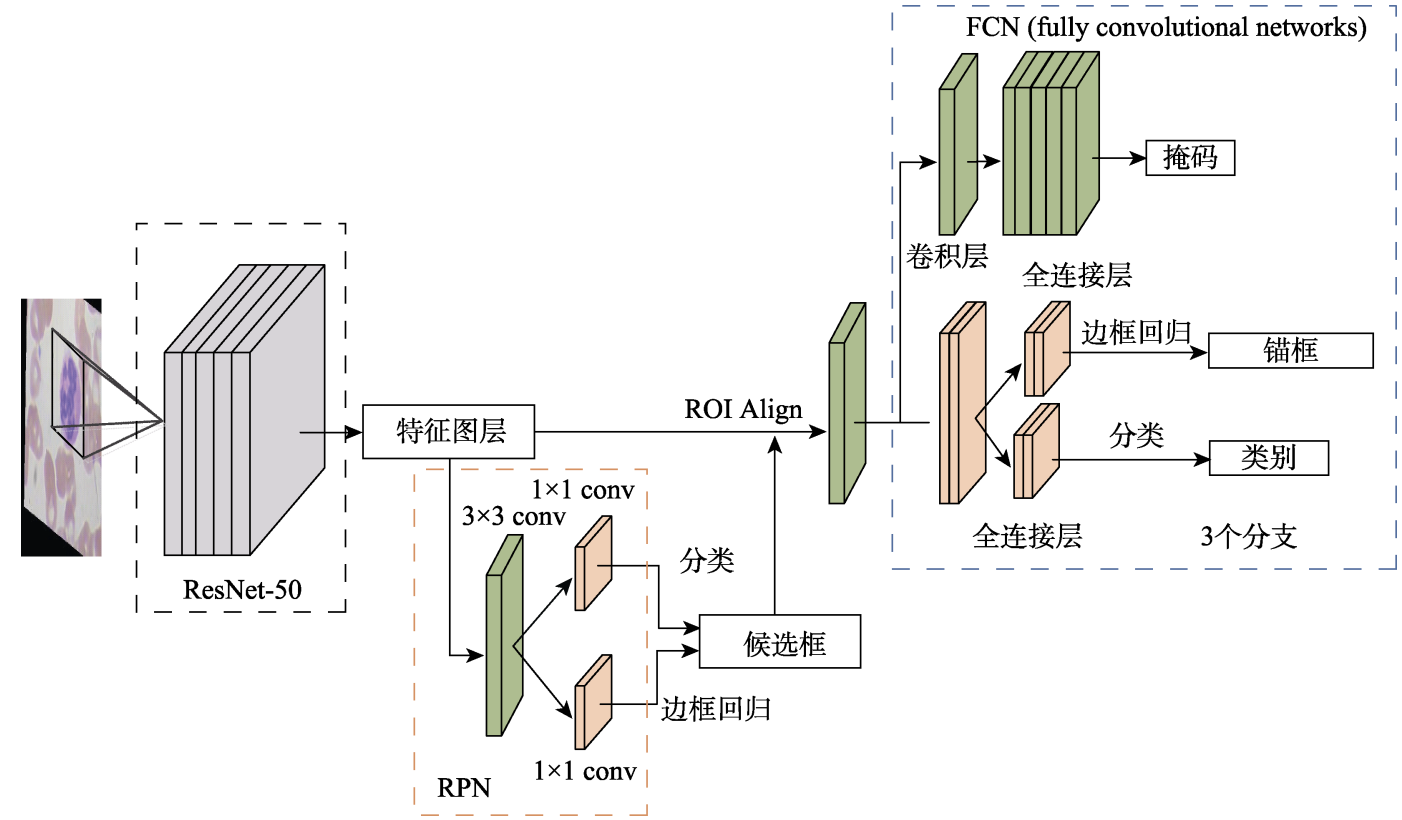

图 1 Mask R-CNN 结构示意图

人的差别, 即 $F(x)=H(x)-x$, 其直接将输人信息 传到输出，保护了信息的完整性，简化了学习目标 和难度. 残差函数 $F(x)$ 使用 3 个卷积层, 其中卷 积核大小分别为 $1 \times 1,3 \times 3$ 和 $1 \times 1$, 中间的 $3 \times 3$ 卷 积在第 1 个降维 $1 \times 1$ 卷积层下减少了计算量，在第 2 个 $1 \times 1$ 卷积层下做了还原, 既保持了精度又减少 了计算量.
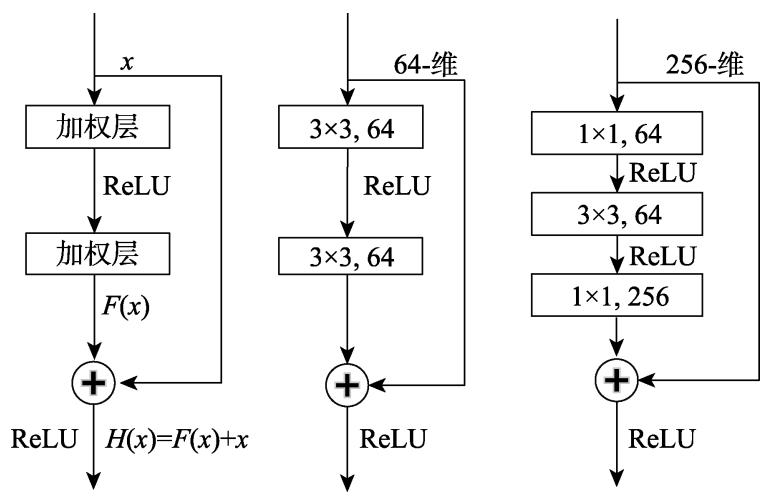

图 2 基础残差块结构

\section{3 特征金字塔网络}

特征金字塔网络 (feature pyramid networks, FPN $)^{[24]}$ 可以融合浅层和深层特征，获得更加鲁棒 的语义信息. 利用浅层特征可以将简单的目标区分 开，利用深层特征将复杂的目标区分开. ResNet-50 能够提取代表白细胞的特征，使用 FPN 模块可以 对 ResNet-50 提取到的多尺度特征进行融合，以获 得更具有代表性和可靠性的特征，从而提高网络 性能.
FPN 分为自下而上的路径、自上而下的路径和 横向连接 3 个部分, 如图 3 所示. 在自下而上的路径 中, 白细胞图像依次经过 5 个阶段, 分别为 conv1, conv2, conv3, conv4, conv5. ResNet-50使用每个阶段 的最后一个残差结构的特征激活作为输出, 将这 些残差模块输出表示为 $\left\{C_{1}, C_{2}, C_{3}, C_{4}, C_{5}\right\} .\left\{C_{2}\right.$, $\left.C_{3}, C_{4}, C_{5}\right\}$ 相对输人图像具有 $\{4,8,16,32\}$ 像素的步 长, $C_{2}, C_{3}, C_{4}$ 和 $C_{5}$ 之后连接 $1 \times 1 \times 256$ 的卷积, 生 成通道数均为 256 的特征层 $M_{2}, M_{3}, M_{4}$ 和 $M_{5}$. 自 上而下的路径中 $M_{5}$ 做 2 倍上采样后与 $M_{4}$ 相加; 再 用 $3 \times 3$ 卷积核 (为了消除上采样的混叠效应)处理已 经融合的特征图, 以生成最后需要的特征图 $P_{4}$. 依 此类推, $P_{3}=M_{3}+2 \times M_{4}, P_{2}=M_{2}+2 \times M_{3}, P_{5}$ 是由
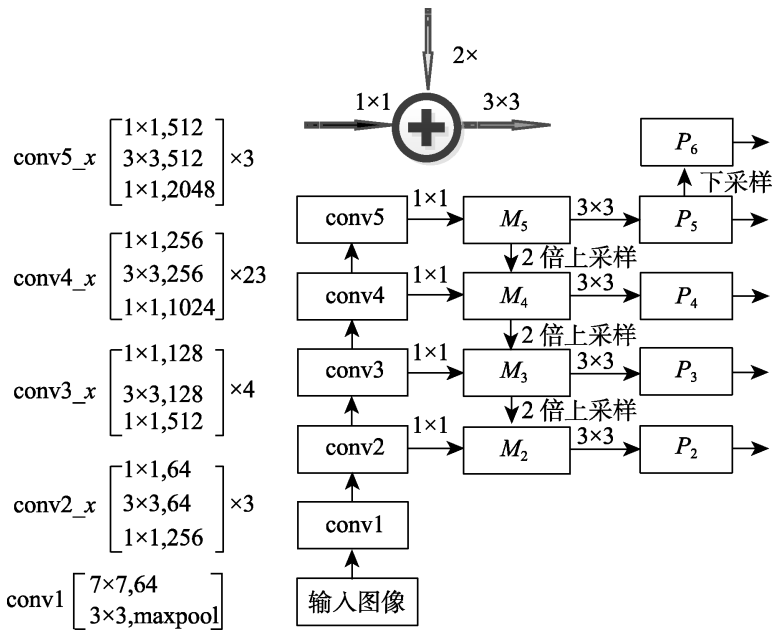

图 3 FPN 结构 
$M_{5}$ 单独经过 $3 \times 3$ 的卷积层生成, $P_{6}$ 为 $P_{5}$ 经过下 采样之后形成的特征层.

\section{2 本文方法}

在复现 Mask R-CNN过程中, 发现 Mask R-CNN 对白细胞进行检测和分割时存在以下缺陷：(1) 因 为缺少底层信息而出现漏检和分类错误的现象; (2) 因为缺少边缘信息而没有完全分割出白细胞 轮廓边缘. 为此, 本文改进了原始 Mask R-CNN, 提出了 C-Mask(Cell-Mask)网络模型. 针对缺陷(1), C-Mask 网络结合特征融合模块对底层和顶层特征 进行多尺度融合; 针对缺陷(2), C-Mask 网络结合 通道空间模块重新标定特征，提升了白细胞检测 的精度.

\section{1 跳跃连接}

因为缺少底层特征信息，导致白细胞出现漏 检和分类错误问题. 针对此问题, 本文提出了 Skip-FPN 模块作为面向白细胞特征融合的模块, 融合更多可靠的、可使用的特征，如图 4 所示.

在 FPN 算法中，在自底向上的过程中，底层 特征传递到顶层需要经过 conv1, conv2, conv3, conv4, conv5 中 50 个网络层的传递，底层信息丢失 严重, 虽然 FPN 中 $P_{5}$ 已经间接获得了底层的特征, 但是流动线太长 ${ }^{[21]}$. 因此, 本文提出了 Skip-FPN 模块, 将 conv2 的最后一个残差结构的特征命名为 $N_{2}$, 然后进行步长为 2 的上采样生成 $N_{3}$, 同理生
成 $N_{4}$ 和 $N_{5} . N_{5}$ 之后连接一个 $1 \times 1 \times 256$ 的卷积层, 生成和 $P_{5}$ 同样的通道数并进行相加, 这样底层特 征就经过 3 层网络层传递到顶层，能够加速获得更 多底层特征信息，提升白细胞检测的平均精度.

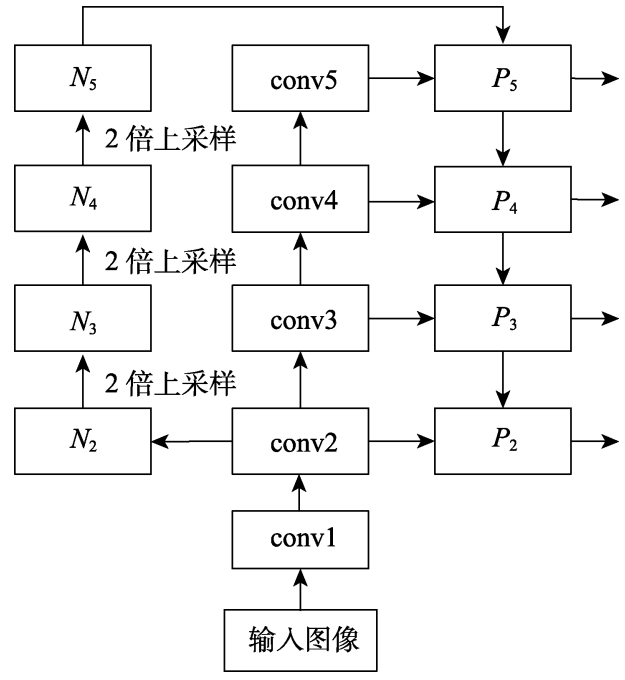

图 4 Skip-FPN 模块结构

\section{2 通道空间加权模块}

本文将通道空间加权模块与 FPN 相结合，提 出了通道空间加权特征金字塔网络(channel spatial feature pyramid networks, CSFPN)模块, 如图 5 所示.

第 1 部分为 ResNet-50 基础网络, 包含 5 个阶 段，每个阶段的最后一个残差结构的特征激活作 为输出, 表示为 $\left\{C_{1}, C_{2}, C_{3}, C_{4}, C_{5}\right\}, C_{2}$ 输出大小为 $256 \times 256 \times 256, C_{3}$ 输出大小为 $128 \times 128 \times 512, C_{4}$

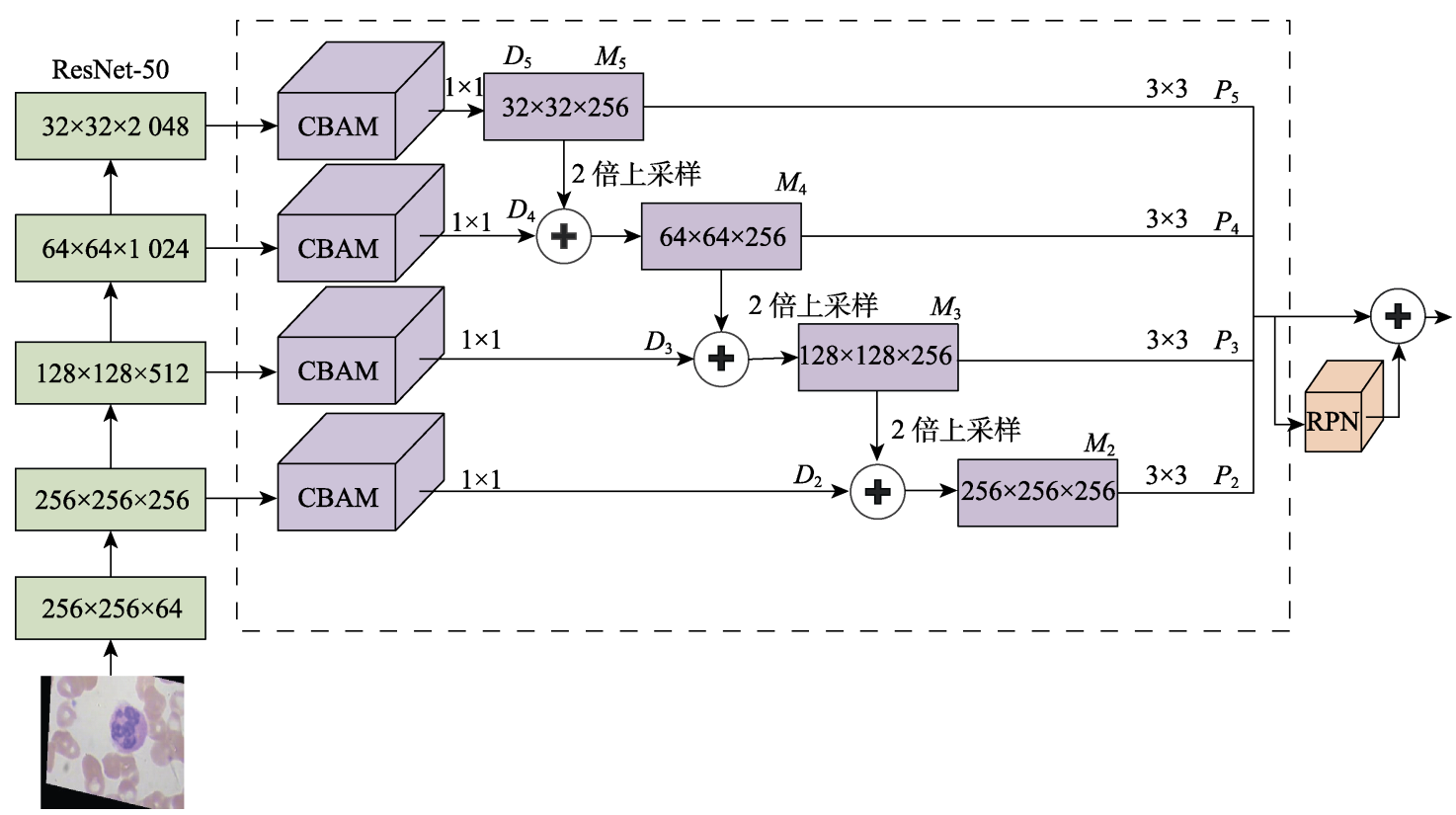

图 5 CSFPN 结构 
输出大小为 $64 \times 64 \times 1024, C_{5}$ 输出大小为 $32 \times 32 \times$ 2048 . 第 2 部分为本文提出的 CSFPN 模块, 由于 网络浅层提取的特征包含许多细节, 深层的特征 更加抽象, 利用 CSFPN 模块对生成的特征进行通 道和空间加权, 以学习的方式对特征重新标定, 增 强有效的特征，抑制无用的特征. 如图 5 所示，将 $C_{2}$ 输出大小为 $256 \times 256 \times 256$ 的特征图 $F$ 输人卷 积注意力机制模块 (convolutional block attention module, $\mathrm{CBAM}$ )中, 首先将输人特征图 $F$ 在空间 维度上进行压缩，分别进行一个空间的全局平均 池化和最大池化，生成 2 个 $1 \times 1 \times 256$ 的通道描述 $F_{\mathrm{avg}}^{\mathrm{s}}$ 和 $F_{\text {max }}^{\mathrm{s}}$, 以聚合特征映射的空间信息, 再分 别送人一个 2 层的神经网络, 核心为 $1 \times 1$ 卷积核, 进行信息的提取, 第 1 层神经元个数为 $256 / 16$, 第 2 层神经元个数为 256. 之后经过一个 Sigmoid 函 数得到通道特征, 即

$$
\begin{aligned}
& M_{\mathrm{c}}(F)=\delta(\operatorname{MLP}(\operatorname{avg}(F))+\operatorname{MLP}(\max (F)))= \\
& \delta\left(W_{1}\left(W_{0}\left(F_{\text {avg }}^{\mathrm{s}}\right)\right)+W_{1}\left(W_{0}\left(F_{\max }^{\mathrm{s}}\right)\right)\right)
\end{aligned}
$$

其中, $W_{0} \in \mathbb{R}^{C / r}, W_{1} \in \mathbb{R}^{C}$; MLP 共享全连接层; $F_{\text {avg }}^{\mathrm{s}}$ 和 $F_{\text {max }}^{\mathrm{s}}$ 分别表示平均池化和最大池化; $\delta$ 表 示 Sigmoid 操作; $r$ 表示缩放率.

其次，通道特征图进行基于通道的全局最大 池化和平均池化得到 2 个 $256 \times 256 \times 1$ 的通道描述 $F_{\mathrm{avg}}^{\mathrm{s}}$ 和 $F_{\max }^{\mathrm{s}}$, 之后基于通道做相加操作, 再经过 一个卷积核为 $7 \times 7$ 的卷积操作, 降维为一个通道, 最后经过 Sigmoid 函数生成空间注意力特征, 即

$$
\begin{aligned}
M_{\mathrm{s}}(F)= & \delta\left(f^{7 \times 7}([\operatorname{avg}(F) ; \max (F)])\right)= \\
& \delta\left(f^{7 \times 7}\left(\left[F_{\text {avg }}^{\mathrm{s}} ; F_{\max }^{\mathrm{s}}\right]\right)\right)
\end{aligned}
$$

其中, $\delta$ 为 Sigmoid操作; $f^{7 \times 7}$ 表示卷积核的大小; $F_{\text {avg }}^{\mathrm{s}}$ 和 $F_{\text {max }}^{\mathrm{s}}$ 表示为平均池化和最大池化.

以此类推, $C_{3}, C_{4}$ 和 $C_{5}$ 模块特征图分别输人 $\mathrm{CBAM}^{[22]}$ 中生成注意力特征图. $\mathrm{CBAM}$ 之后连接一 个 $1 \times 1 \times 256$ 卷积层, 使输出特征图拥有相同的通 道数以进行多尺度特征融合. 在多尺度特征融合 过程中， $D_{5}$ 通过上采样，再加上(特征图中每个相 同位置元素直接相加). $D_{4}$ 经过 $1 \times 1$ 卷积后的特征 图, 得到 $M_{4}$. 同理得到 $M_{3}$ 和 $M_{2} . M$ 层特征图 再经过 $3 \times 3$ 卷积(减轻最近邻插值带来的混叠影 响, 周围的范围都相同), 得到最终的 $P_{2}, P_{3}, P_{4}, P_{5}$ 层特征.

CBAM 利用卷积层后特征图的全局信息动态
地对通道和空间的依赖性进行建模, 以提升网络 的特征学习能力. 该模块沿着通道和空间 2 个独立 的维度, 使网络学习到重要特征的同时, 压缩不必 要的特征, 让网络依照特征的重要程度有选择性 地进行优化. CBAM 结构如图 6 所示, 特征图经过 通道维度计算其注意力图, 然后将注意力图与输 人的特征图相乘形成 $F^{\prime}$ 进行特征的自适应学习; 随后, 经过空间维度计算其注意力图, 并将注意力 图与输人的特征图相乘形成新的特征图.

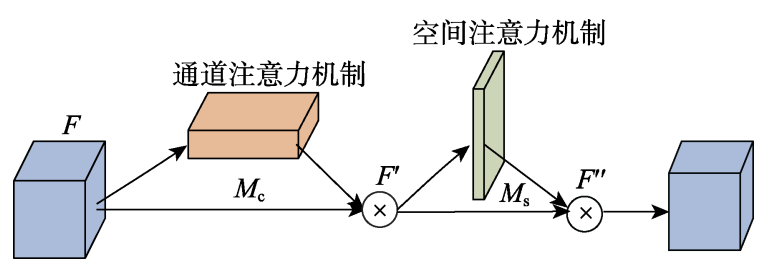

图 $6 \mathrm{CBAM}$ 结构

\section{3 实 验}

本节通过实验果以验证所提出的白细胞检测 模型的性能. 首先介绍数据集及评估标准; 然后将 本文方法实验结果与其他方法进行比较, 证明在 数据集上达到了较高的分割准确率.

\section{1 数据集及评估指标}

数据集从 Kaggle 上获取. 该数据集包含 12500 幅血细胞图像, 分为 4 类细胞, 分别为嗜酸性粒细 胞、淋巴细胞、单核细胞和嗜中性粒细胞. 本文根 据样本特征代替整体特征的思想, 选取 4000 幅样 本数据代表 12500 幅整体数据, 以此用来网络的 训练和测试. 该 4000 幅图像包含上述 4 类白细胞 的所有形态, 每类分别有 1000 幅图像, 每幅图像 尺寸为 $320 \times 240$ 像素.

为了更好地定量分析分割结果, 采取以下量 化指标: (1) 准确率 $p$; (2) 召回率 $r$, 表示测试集中 所有白细胞样例中被正确识别为白细胞的比例; (3) 真实值和预测值 2 个集合的交并比(mean intersection over union, mIoU); (4) 平均精确度 (average precision, AP), 即准确率-召回率曲线下 面的面积, 通常, 一个越好的分类器, AP 值越高; (5) 平均 $\mathrm{AP}$ (mean average precision, $\mathrm{mAP}$ ), 是多个 类别 $\mathrm{AP}$ 的平均值, $\mathrm{mAP}$ 的大小为 $0 \sim 1$, 越大越好; (6) Dice 系数, 一种集合相似度度量指标, 用于计 算预测结果和标定结果 2 个样本的相似度; (7) 反 正例率(false positive rate, FPR), 即把非白细胞预 测为白细胞的实例占所有白细胞实例的百分比. 
各指标具体计算公式为

$$
\begin{gathered}
p=\frac{T}{T+F} \\
r=\frac{T}{T+M} \\
\mathrm{IoU}=\frac{T}{F+M+T} \\
\mathrm{AP}=\int_{0}^{1} p(r) \mathrm{d} r \\
\text { Dice }=\frac{2 T}{F+2 T+M} \\
\mathrm{FPR}=\frac{F}{F+N}
\end{gathered}
$$

其中, $T$ 为白细胞被准确识别为白细胞的像素点; $N$ 为非白细胞被正确识别为非白细胞的像素点; $F$ 为 非白细胞被错误识别为白细胞的像素点; $M$ 为白细 胞被错误识别为非白细胞的像素点.

\section{2 实验结果及分析}

本实验选取的 4000 幅图像中, 3200 幅图像用 作训练, 400 幅图像用作验证, 400 幅图像用作测试. 训练中 batch_size 设置为 8, 动量设置为 0.9 , 初始 学习率为 $1 \times 10^{-3}$, 权重衰减为 $2 \times 10^{-4}$, 训练过程 持续了 40 个 epoch. 采用测试图像定量评价的平均 值作为定量分析的结果. 结果分析包括网络训练 过程分析、C-Mask 与 Mask R-CNN 对比分析以及 C-Mask 与其他分割方法对比分析 3 个方面，下面 将依次进行阐述.

图 7 所示为训练过程的 loss 曲线, 可知训练时 loss 曲线收玫较快、较平滑, 在 epoch 为 35 时趋于 平缓，基本持平.

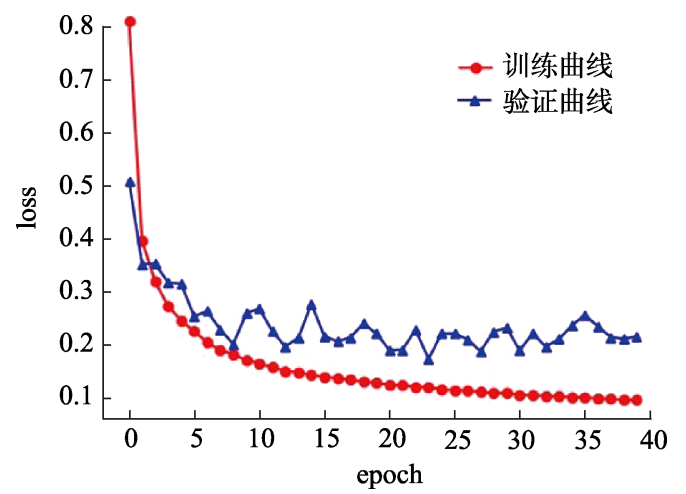

图 7 训练过程的 loss 曲线

\subsection{1 本文方法与 Mask R-CNN 比较}

为了验证改进网络的有效性，本节利用 $\mathrm{mAP}$, 平均准确率 $p_{\mathrm{m}}$, 平均召回率 $r_{\mathrm{m}}$ 和 $\mathrm{mIoU}$ 这 4 个指 标对比改进前后网络的性能, 如表 1 所示. Mask R-CNN 采用的是 FPN 特征融合方式, 本文方法采 用的是通道空间加权和 Skip-FPN 多尺度特征融合
方法, 由表 1 中数据可以看出, 本文方法在 4 个性 能方面均高于 Mask R-CNN.

表 12 种模型结果对比

\begin{tabular}{lcccc}
\hline \multicolumn{1}{c}{ 方法 } & $\mathrm{mAP}$ & $p_{\mathrm{m}}$ & $r_{\mathrm{m}}$ & $\mathrm{mIoU}$ \\
\hline Mask R-CNN $^{[19]}$ & 0.9700 & 0.6483 & 0.6575 & 0.8910 \\
C-Mask & 0.9825 & 0.6550 & 0.6608 & 0.8930 \\
\hline
\end{tabular}

本文方法结合通道间空间加权的多尺度特征 融合从细胞轮廓边缘、细胞分类错误、漏检率等 3 个方面与 Mask R-CNN 进行结果的比较, 如表 2 所 示. 从示例 1 中的 Mask R-CNN 图像的黑框中可以 看出算法并没有完全分割出白细胞轮廓边缘，而 C-Mask 图像的黑框中可以看出完整地分割出了白 细胞的边缘. 从示例 2 中 Mask R-CNN 图像的黑框 中可以看出检测结果出现分类错误现象, 嗜酸性 粒细胞被错误识别成中性粒细胞, 而本文方法正 确识别出是嗜酸性粒细胞. Mask R-CNN 对白细胞 的 FPR 为 $3.00 \%$, 本文方法对白细胞的 FPR 为 $1.75 \%$ ，网络性能得到提高. 示例 3 中 Mask R-CNN 的检测结果中会出现漏检的现象, 而本文方法对 白细胞检测率达到 $100.00 \%$.

\section{表 2 Mask R-CNN 与本文方法对比}

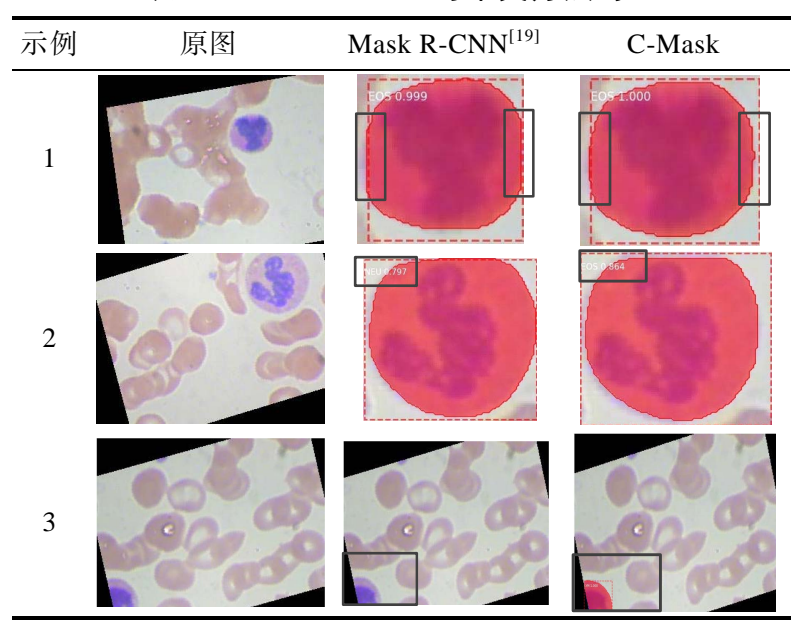

\subsection{2 本文方法与其他方法比较}

为了展示 C-Mask方法的性能, 本文采用 $\mathrm{mAP}$, mIoU, FPR 和 Dice 这 4 个指标对比分析本文方法 与 4 个代表性深度学习网络的性能, 对比实验采用 统一的数据集训练模型, 测试如表 3 所示. 全卷积 网络(fully convolutional networks, FCN) ${ }^{[25]}$, U-Net, SegNet 等网络的分割方法是在整幅图像上进行分 割, 很容易受到血涂片中杂质的影响, 预测会受到 红细胞和一些额外的染色质的干扰, 会降低最终 的分割结果的准确性. 而本文方法仅对 ROI Align 层定位的 ROI进行分割, 这样缩小分割范围, 从而 
使 mIoU 值对于 FCN, U-Net, SegNet 分别提高了 0.2082，0.062 9，0.1360. FCN 忽略了高分辨率的特 征图导致边缘信息的丢失, SegNet 和 U-Net 因为多 次上采样和下采样，导致信息在不同程度上丢失 了, 因此其分割准确率较低. 针对以上问题，本文 结合通道空间加权与多尺度特征高了 0.2615. 因 此, 本文方法在 4 融合的方法对白细胞进行检测和 分割, 有效融合底层细节信息和高层抽象信息, 从 而使 mAP 值提个性能方面都是最优的, 具有良好 的分割效果.
表 3 不同方法的评估指标和结果

\begin{tabular}{lcccc}
\hline \multicolumn{1}{c}{ 方法 } & mAP & mIoU & FPR & Dice \\
\hline FCN $^{[25]}$ & 0.7210 & 0.6848 & 0.0375 & 0.8129 \\
SegNet $^{[16]}$ & 0.7911 & 0.7570 & 0.2089 & 0.8616 \\
U-Net $^{[17]}$ & 0.8680 & 0.8301 & 0.1320 & 0.9071 \\
DeepLabv3+ $^{[26]}$ & 0.7466 & 0.7058 & 0.2534 & 0.8275 \\
Mask R-CNN & \\
C-Mask & 0.9700 & 0.8910 & 0.0300 & 0.9423 \\
\hline
\end{tabular}

表 4 展示了本文方法与其他方法的分割结果 示意图.

表 4 不同网络分割结果图

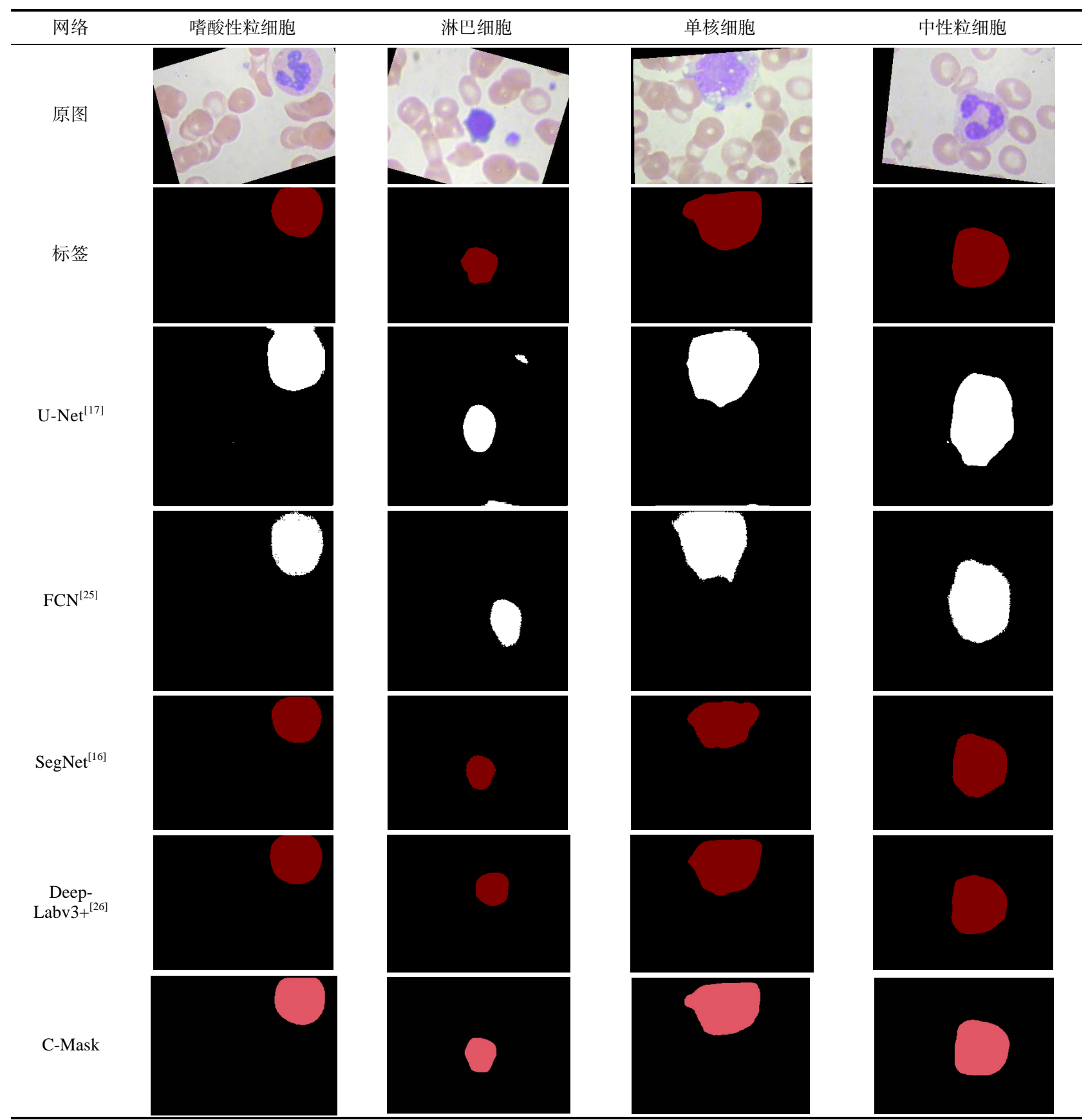


从表 4 可以看出, U-Net 测试结果中的淋巴细 胞受到了杂质的影响, 而且分割的白细胞形态有 偏差. FCN 和 SegNet 测试结果中白细胞边缘有毛 刺、不光滑, 分割的白细胞形态有偏差. 本文方法 完整分割白细胞形态且分割细胞边缘平均精度更 高. DeepLabv3+只是语义分割, 且分割的淋巴细胞 有形态偏差，本文方法可以对白细胞进行实例分 割, 分割的结果更好. 本文方法成功的原因主要有 以下几点: (1) 使用了 Skip-FPN 模块, 缩短了底层 特征到高层特征的路径, 能够使底层信息更快地 传递到高层上, 融合更多白细胞细节信息; (2) 加 人了 CSFPN, 通过分析学习特征图的不同通道像 素的重要程度和同一个通道不同位置像素的重要 程度增强特征融合.

\section{4 结 语}

本文提出了以 Mask R-CNN 为基础进行改进 的 C-Mask 网络, 实现了端到端的白细胞定位、分 类和分割. 针对白细胞出现漏检、分类错误和没有 完整分割细胞形态等问题，本文提出的 C-Mask 网 络结合通道空间加权模块和 Skip-FPN 模块进行多 尺度特征融合，融合了不同通道的重要特征和同 一通道不同位置的重要特征，对特征进行重新标 定，缩短底层特征到顶层特征之间的距离，使白细 胞检测的平均精度达到了 $98.25 \%$. 实验结果和分 析表明，本文方法比 Mask R-CNN 和其他网络的分 割精度更高，检测性能更好. 未来的工作将收集包 含更多白细胞种类的医学图像数据集和骨髓中白 细胞图像数据集, 对本文提出的网络进行评估, 实 现在各种环境下进行白细胞检测与分割。

\section{参考文献(References):}

[1] Wang S T, Wang M. A new detection algorithm (NDA) based on fuzzy cellular neural networks for white blood cell detection[J]. IEEE Transactions on Information Technology in Biomedicine, 2006, 10(1): 5-10

[2] Liu H, Cao H C, Song E M, et al. Bone marrow cells detection: a technique for the microscopic image analysis[J]. Journal of Medical Systems, 2019, 43(4): Article No.82

[3] Salem N M. Segmentation of white blood cells from microscopic images using $\mathrm{K}$-means clustering[C] //Proceedings of the 31st National Radio Science Conference. Los Alamitos: IEEE Computer Society Press, 2014: 371-376

[4] Rezatofighi S H, Soltanian-Zadeh H. Automatic recognition of five types of white blood cells in peripheral blood[J]. Comput- erized Medical Imaging and Graphics, 2011, 35(4): 333-343

[5] Liu Z, Liu J, Xiao X Y, et al. Segmentation of white blood cells through nucleus mark watershed operations and mean shift clustering[J]. Sensors, 2015, 15(9): 22561-22586

[6] Duan J, Yu L. A WBC segmentation method based on HSI color space[C] //Proceedings of the 4th IEEE International Conference on Broadband Network and Multimedia Technology. Los Alamitos: IEEE Computer Society Press, 2011: 629-632

[7] Cao H C, Liu H, Song E M. A novel algorithm for segmentation of leukocytes in peripheral blood[J]. Biomedical Signal Processing and Control, 2018, 45(8): 10-21

[8] Huang Zhen, Zhao Jianwei, Chu Jianjun, et al. Efficient segmentation algorithm for white blood cell[J]. Computer Engineering and Applications, 2016, 52(21): 188-194(in Chinese) (黄震, 赵建伟, 楚建军, 等. 高效的白细胞分割算法[J]. 计 算机工程与应用, 2016, 52(21): 188-194)

[9] Yang Xiaoqing, Yang Qiuxiang, Yang Jian. Microscopic cell image processing and application based on morphology[J]. Computer Systems Applications, 2016, 25(3): 220-224(in Chinese)

(杨小青, 杨秋翔, 杨剑. 基于形态学的显微细胞图像处理 与应用[J]. 计算机系统应用, 2016, 25(3): 220-224)

[10] Mohammadi E, Orooji M. An unsupervised and supervised combined approach for white blood cells segmentation[C] //Proceedings of the 25th National and the 3rd International Iranian Conference on Biomedical Engineering. Los Alamitos: IEEE Computer Society Press, 2018: 209-214

[11] Loddo A, Putzu L, Ruberto C D, et al. A computer-aided system for differential count from peripheral blood cell images[C] //Proceedings of the 12th International Conference on Signal Image Technology and Internet-Based Systems. Los Alamitos: IEEE Computer Society Press, 2016: 112-118

[12] Razzak M I, Naz S. Microscopic blood smear segmentation and classification using deep contour aware CNN and extreme machine learning[C] //Proceedings of the 30th IEEE Conference on Computer Vision and Pattern Recognition Workshops. Los Alamitos: IEEE Computer Society Press, 2017: 801-807

[13] Qin F W, Gao N N, Peng Y, et al. Fine-grained leukocyte classification with deep residual learning for microscopic images[J]. Computer Methods and Programs in Biomedicine, 2018, 162(8): 243-252

[14] Tiwari P, Qian J, Li Q C, et al. Detection of subtype blood cells using deep learning[J]. Cognitive Systems Research, 2018, 52(12): 1036-1044

[15] Yu W, Chang J, Yang C, et al. Automatic classification of leukocytes using deep neural network[C] //Proceedings of the 12th IEEE International Conference on Advanced Semiconductor Integrated Circuits. Los Alamitos: IEEE Computer Society Press, 2017: 1041-1044

[16] Tran T, Kwon O H, Kwon K R, et al. Blood cell images segmentation using deep learning semantic segmentation[C] //Proceedings of the IEEE International Conference on Electronics and Communication Engineering. Los Alamitos: IEEE Computer Society Press, 2018: 13-16

[17] Zhang X Q, Zhao S G. Blood cell image classification based on 
image segmentation preprocessing and CapsNet network model[J]. Journal of Medical Imaging and Health Informatics, 2019, 9(1): 159-166

[18] Wang Q W, Bi S S, Sun M L, et al. Deep learning approach to peripheral leukocyte recognition[J]. PLoS One, 2019, 14(6): Article No.e0218808

[19] He K M, Gkioxari G, Dollár P, et al. Mask R-CNN[J]. IEEE Transactions on Pattern Analysis and Machine Intelligence, 2020, 42(2): 386-397

[20] Ren S Q, He K M, Girshick R. Faster R-CNN: towards real-time object detection with region proposal networks[J]. IEEE Transactions on Pattern Analysis and Machine Intelligence, 2017, 39(6): 1137-1149

[21] Liu S, Qi L, Qin H F, et al. Path aggregation network for instance segmentation[C] //Proceedings of the 31st IEEE/CVF Conference on Computer Vision and Pattern Recognition. Los Alamitos: IEEE Computer Society Press, 2018: 8759-8768

[22] Woo S, Park J, Lee J Y, et al. CBAM: convolutional block at- tention module[C] //Proceedings of the 15th European Conference on Computer Vision. Heidelberg: Springer, 2018: 3-19

[23] He K M, Zhang X Y, Ren S Q, et al. Deep residual learning for image recognition[C] //Proceedings of the IEEE Conference on Computer Vision and Pattern Recognition. Los Alamitos: IEEE Computer Society Press, 2016: 770-778

[24] Lin T Y, Dollár P, Girshick R, et al. Feature pyramid networks for object detection[C] //Proceedings of the 30th IEEE Conference on Computer Vision and Pattern Recognition. Los Alamitos: IEEE Computer Society Press, 2017: 936-944

[25] Long J, Shelhamer E, Darrell T. Fully convolutional networks for semantic segmentation[J]. IEEE Transactions on Pattern Analysis and Machine Intelligence, 2014, 39(4): 640-651

[26] Chen L, Zhu Y, Papandreou G, et al. Encoder-decoder with atrous separable convolution for semantic image segmentation[C] //Proceedings of the 15th European Conference on Computer Vision. Los Alamitos: IEEE Computer Society Press, 2018, 11211: 833-851 